\title{
NEW IMPROVEMENT OF THE CONVERSE JENSEN INEQUALITY
}

\section{J. PEČARIĆ AND J. PERIĆ}

Abstract. We give a new refinement of the converse Jensen inequality for linear functionals as well as improvements of some related results. Especially, we give two refinements of the converse Hölder inequality for functionals and a refinement of the converse Minkowski inequality for functionals. Application on the quasi-arithmetic and power mean is given.

Mathematics subject classification (2010): 26D15.

Keywords and phrases: Convex functions, positive linear functional, converse Jensen inequality, converse Hölder inequality, converse Minkowski inequality, quasi-arthmetic mean.

\section{REFERENCES}

[1] P. R. Beesack, Josip E. PeČArić, On Jessen's inequality for convex functions, J. Math. Anal. Appl. 110 (1985), no. 2, 536-552.

[2] P. R. BeEs Ack, Josip E. PeČArIĆ, On Knopp's inequality for convex functions, Canad. Math. Bull. 30 (1987), no. 3, 267-272.

[3] D. ChOI, M. KRniĆ, J. PeČARIĆ, Improved Jensen-type inequalities via linear interpolation and applications, Math. Inequal. 11 (2017), 301-322.

[4] D. ChOI, M. KRnić, J. PeČARIĆ, More accurate classes of Jensen-type inequalities for convex and operator convex functions, submitted for publication.

[5] T. Furuta, J. Mićić, J. PeČArić, J. Seo, Mond-Pečarić Method in Operator Inequalities / Inequalities for bounded selfadjoint operators on a Hilbert space, Element, Zagreb, 2005.

[6] J. L. W. V. Jensen, Om konvexe funktioner og uligheder mellem Middelvaerdier, (German) Nyt. Tidsskrift for Mathematik 16 B (1905), 49-69.

[7] B. JESSEN, Bemaerkinger om konvekse Funktioner og Uligheder imellem Middelvaerdier I, Mat. Tidsskrift B (1931), 17-29.

[8] M. Klaričić Bakula, J. Pečarić, J. Perić, On the converse Jensen inequality, Appl. Math. Comput. 218 (11) (2012), 6566-6575.

[9] K. KNOPP, Über die maximalen Abstände und Verhältnisse verschiedener Mittelwerte, (German) Math. Z. 39 (1935), no. 1, 768-776.

[10] P. LAh, M. Ribarič, Converse of Jensen's inequality for convex functions, Univ. Beograd Publ. Elektrotehn. Fak. Ser. Mat. Fiz. no. 412-460 (1973), 201-205.

[11] D. S. Mitrinović, J. E. PeČArić, A. M. Fink, Classical and new inequalities in analysis, Mathematics and its Applications (East European Series), 61, Kluwer Academic Publishers Group, Dordrecht, 1993.

[12] Josip E. PeČarić, Frank Proschan, Y. L. Tong, Convex functions, partial orderings, and statistical applications, Mathematics in Science and Engineering, 187, Academic Press, Inc., Boston, MA, 1992.

[13] J. PeČArić, J. Perić, S. VARošAnEC, Refinements of the converse Hölder and Minkowski inequalities, submitted for publication. 